\title{
Carbide Formation upon Tempering at Low Temperatures in Fe-Mn-C Alloys
}

\author{
By Yasuji Tanaka* and Ken'ichi Shimizu**
}

\begin{abstract}
Processes of the carbide formation upon low temperature tempering in Fe-4.1 mass $\% \mathrm{Mn}-1.25$ mass $\% \mathrm{C}$ and $\mathrm{Fe}-5.2$ mass $\% \mathrm{Mn}-0.93$ mass $\% \mathrm{C}$ alloys have been examined by means of $\mathrm{X}$-ray and electron diffraction and electron microscopy. Upon the tempering at $358 \mathrm{~K}$, the martensite lattice parameters change in two stages (the preliminary and the first stages according to the former definition) as a function of the tempering period of time. In the preliminary stage, a long period ordered tetragonal phase, being a modification of $\mathrm{Fe}_{4} \mathrm{C}$ carbide, is formed as found by Nagakura et al. in $\mathrm{Fe}-\mathrm{C}$ alloys. Another diffraction effect suggesting the formation of a modulated structure and/or a short range order of carbon atoms has also been observed. The so-called intermediate carbide in the first stage is identical to " $\eta$-carbide" found by Hirotsu et al. in $\mathrm{Fe}-\mathrm{C}$ alloys, exhibiting an orthorhombic structure and superlattice reflections due to carbon atom ordering in diffraction patterns. The lattice parameters of the $\eta$-carbide are $a=0.470 \mathrm{~nm}, b=0.429 \mathrm{~nm}$ and $c=0.285 \mathrm{~nm}$, and the orientation relationship to the martensite matrix is $(011)_{M} / /(010)_{\eta},[100]_{M} / /[001]_{\eta}$. The shape of carbide is a very fine disk or ribbon whose largest areal plane is parallel to the $\{100\}_{M}$ or $\{011\}_{M}$ plane, and the carbides parallel to the $\{100\}_{M}$ and $\{011\}_{M}$ planes are arranged along the $\langle 100\rangle_{M}$ and $[001]_{M}$ directions, respectively, forming an apparent rod or plate shape parallel to the $\{100\}_{M}$ or $(001)_{M}$ plane.
\end{abstract}

(Received June 15, 1981)

\section{Introduction}

The present authors have carried out a series of studies on the morphology and crystallography of martensites in the $\mathrm{Fe}-\mathrm{Mn}-\mathrm{C}$ alloys $^{(1)}-{ }^{(6)}$. In the last X-ray diffraction study on austenite and martensites lattice parameters, it was found that the $c$ parameters of martensites in those alloys were reduced upon ageing at room temperature while the $a$ parameters were not. Such a reduction only in the $c$ parameters was attributed to a getting away phenomenon of carbon atoms from the octahedral sites along the $[001]_{M}$ axis to form a segregate or a superlattice $^{(5)}$. It is of great interest to know details of such a carbon segregation or a superlattice formation as well as the carbide formation in the $\mathrm{Fe}-\mathrm{Mn}-\mathrm{C}$ alloys.

Many studies have so far been carried out on the carbide formation upon tempering of martensites in quenched $\mathrm{Fe}-\mathrm{C}$ and $\mathrm{Fe}-(\mathrm{Ni}$ or $\mathrm{Cr})-\mathrm{C}$ alloys ${ }^{(7)-(16)}$. According to those studies,

* Graduate School, Osaka University, Suita, Osaka 565, Japan.

** Institute of Scientific and Industrial Research, Osaka University, Yamada-oka, Suita, Osaka 565, Japan. the tempering process can be divided into three stages, the first, second and third, corresponding to the formation of an intermediate carbide, the decomposition of austenite, and the formation of cementite, respectively. Some recent studies, however, have pointed out the existence of a preliminary stage prior to the first stage, and some questions have remained as to the nature of carbon atom segregation in the preliminary stage and the crystal structure of intermediate carbide in the first stage. According to studies by Izotov et al. ${ }^{(17)}$ and Nagakura et al. ${ }^{(18)-(20)}$ using $\mathrm{Fe}-\mathrm{Cr}-\mathrm{C}$ and $\mathrm{Fe}-\mathrm{C}$ alloys, respectively, a carbide $\left(\mathrm{Fe}_{4} \mathrm{C}\right)$ and/or superlattice formation and a modulation and/or a short range ordering of carbon atoms occur in the preliminary stage. As to the intermediate carbide in the first tempering stage, its crystal structure has been known to have a hexagonal lattice, and many workers have named it " $\varepsilon$-carbide" for a long time ${ }^{(12)}$. However, Hirotsu et al..$^{(21)-(23)}$ and Shimizu et $a .^{(24)}$ recently identified the intermediate carbide to be of an orthorhombic lattice. Such an orthorhombic distortion of the hexagonal lattice was attributed by Hirotsu et al. to an ordered arrangement of carbon atoms in the carbide, which was especially named as " $\eta$ - 
carbide" by them, although Shimizu et al. could not succeed in detecting superlattice reflections due to the carbon atom ordering.

It is also of great interest to examine whether or not processes of carbide formation similar to the previous ones mentioned above are realized in the $\mathrm{Fe}-\mathrm{Mn}-\mathrm{C}$ alloys. The present work has been carried out with such an object, and the results are described below.

\section{Experimental Procedures}

The $\mathrm{Fe}-4.1$ mass $\% \mathrm{Mn}-1.25$ mass $\% \mathrm{C}$ and $\mathrm{Fe}-5.2$ mass \% Mn-0.93 mass \% $\mathrm{C}$ alloys in the previous works ${ }^{(3)-(6)}$ have been used in the present work, which will be referred to Specimens $\mathrm{A}$ and $\mathrm{B}$, respectively, in this paper. The alloys with higher carbon contents are usefull for the present object, because the superlattice reflections are necessary to be detected if carbon atoms are ordered in martensites and carbide lattices. A description on the method of alloy preparations will be omitted since it was done in the previous papers ${ }^{(3)-(6)}$. After $20 \times 18 \times 4$ $\mathrm{mm}^{3}$ plates and $3 \mathrm{~mm}$ diameter disks with 0.3 mm thickness were made, they were austenitized in silica capsules filled with argon gas and then quenched into water. The quenched plates and disks were immersed in liquid nitrogen for a time and taken out, and then they were subjected to tempering treatments in oil bath kept at 358 and $482 \mathrm{~K}$ for various periods of time. The tempered plates and disks were used for $\mathrm{X}$-ray diffraction and electron microscopy, respectively.

In the X-ray diffraction study, a surface layer $1 \mathrm{~mm}$ thick was removed from the tempered $4 \mathrm{~mm}$ thick plate by chemical and electrolytic polishing in order to avoid any surface effect such as decarburization during the heat treatments. Thin foils for the electron microscopy were prepared from the tempered $0.3 \mathrm{~mm}$ thick disks by jet-polishing and then by normal electropolishing in a solution of $\mathrm{CH}_{3} \mathrm{OH}$ and $\mathrm{H}_{2} \mathrm{SO}_{4}(4: 1)$. Since the decarburization is feared particularly for $0.3 \mathrm{~mm}$ thick disks, other $3 \mathrm{~mm}$ diameter disks were sometimes prepared from the $4 \mathrm{~mm}$ thick plates used for the X-ray diffraction study and devoted to electron microscopy after thinning.

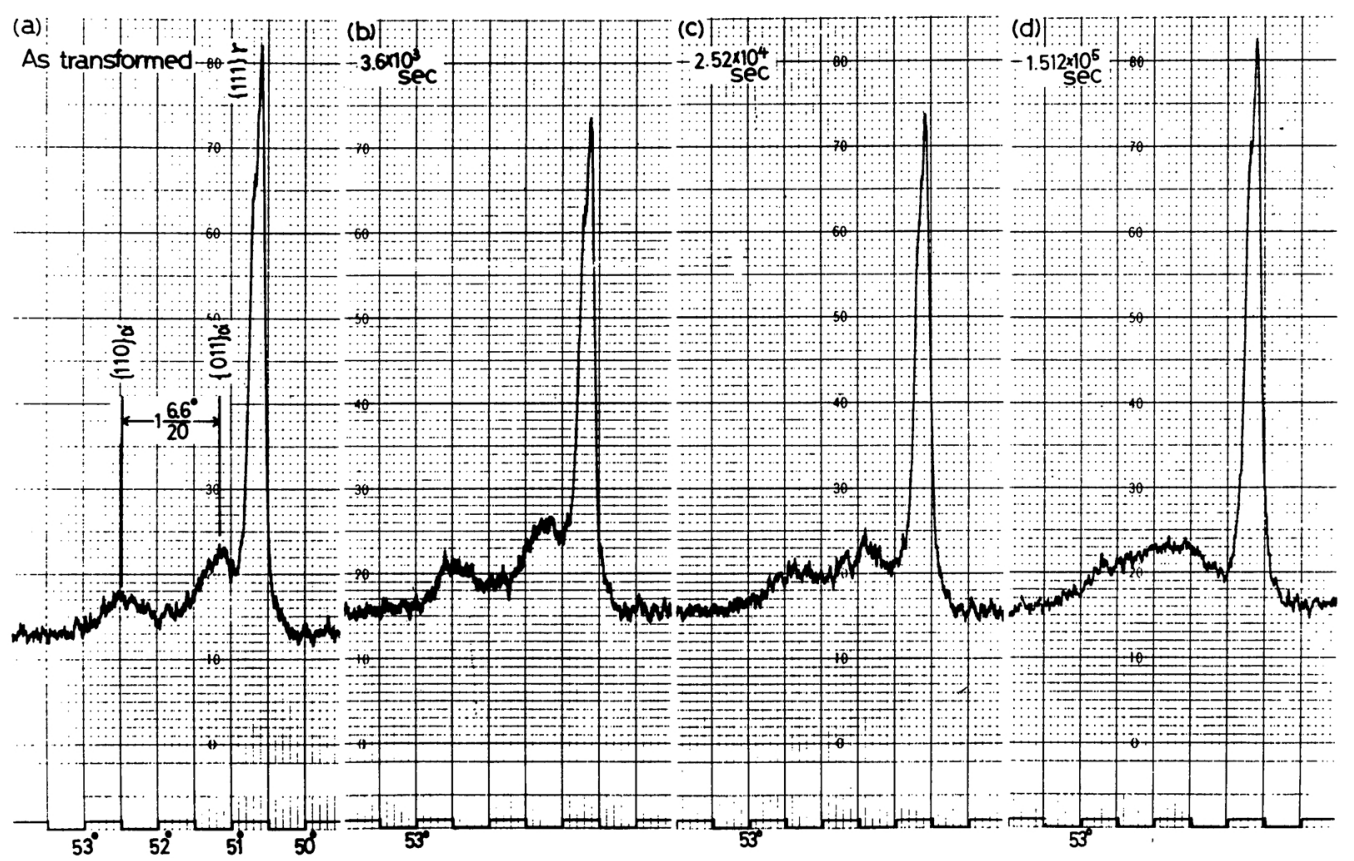

Fig. 1 X-ray diffractometry profiles taken from $\mathrm{Fe}-4.1$ mass $\% \mathrm{Mn}-1.25$ mass $\% \mathrm{C}$ alloy, (a); as-transformed, (b); tempered at $358 \mathrm{~K}$ for $3.6 \mathrm{ks}(1 \mathrm{~h}),(\mathrm{c}) ; 25.2 \mathrm{ks}$ ( $7 \mathrm{~h}$ ), and (d); $151.2 \mathrm{ks}(42 \mathrm{~h})$. They were all taken at room temperature. 
An X-ray diffractometer and an electron microscope used were of a Rigaku Denki D-3F type and of a Hitachi HU-650 type operated at $500 \mathrm{kV}$, respectively. Electron diffraction patterns were recorded on X-ray films in order to detect weak superlattice reflections and to avoid the effect of electron irradiation on the tempering process by shortening the exposure time.

\section{Experimental Results}

\section{X-ray diffraction of martensite lattice parameters}

The $a$ and $c$ parameters and $c / a$ ratios of martensites have been measured by taking $\mathrm{X}$-ray diffractometry profiles. A few examples of the region around the austenite $\{111\}$ peak of the X-ray diffractometry profile taken are shown in Fig. 1, which were from Specimen A tempered at $358 \mathrm{~K}$. (a) is the one from an as-transformed specimen, and (b), (c) and (d) are those from tempered specimens for 3.6, 25.2 and $151.2 \mathrm{ks}(1,7$ and $42 \mathrm{~h})$, respectively. The $\{110\}$ and $\{011\}$ martensite peaks are distinguishable in (a), (b) and (c) but not in (d). The $a$ and $c$ parameters and $c / a$ ratios obtained from such series of X-ray diffractometry profiles for both the alloys are shown in Fig. 2 as a function of the tempering period at 358 $\mathrm{K}$. The lattice parameters and $c / a$ ratios seem to exhibit two kinds of changes, one occurring in the tempering period less than $3.6 \mathrm{ks}$ and the other in the tempering period more than $3.6 \mathrm{ks}$. In the former change, the $c$ parameters and $c / a$ ratios show a little decreasing tendency, although the $a$ parameters are almost constant. In the latter change, however, the $c$ parameters and $c / a$ ratios rapidly decrease and the $a$ parameters slightly increase with increasing tempering period. Such two kinds of changes in the martensite lattice parameters were not observed for the alloys tempered at $428 \mathrm{~K}$. In the case of $428 \mathrm{~K}$ tempering, the $a$ and $c$ parameters were difficult to be distinguished from the beginning of the tempering (within only a few minutes), and therefore the stage corresponding to the former change in $358 \mathrm{~K}$ tempering was not detectable. That is, the lattice

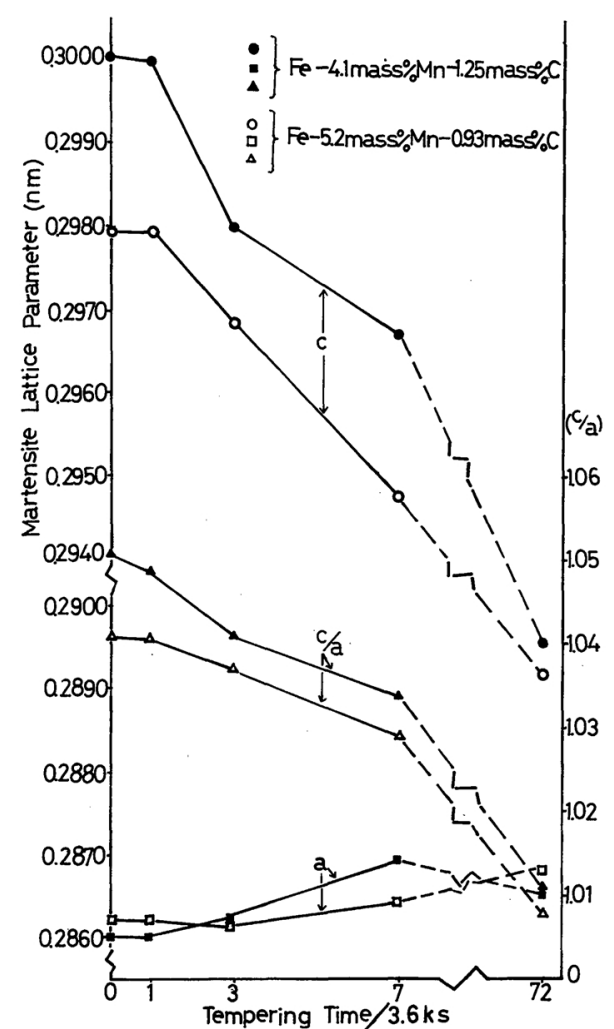

Fig. 2 Martensite lattice parameters and $c / a$ ratios measured as a function of tempering period at $358 \mathrm{~K}$ for $\mathrm{Fe}-4.1$ mass $\% \mathrm{Mn}-1.25$ mass $\% \mathrm{C}$ and $\mathrm{Fe}-5.2$ mass $\% \mathrm{Mn}-0.93$ mass $\% \mathrm{C}$ alloys.

parameters exhibited a rapid change from the beginning and reached a saturated value within a very short period.

The observed decrease in the $c$ parameters and $c / a$ ratios with increasing tempering period may be due to a getting away phenomenon of carbon atoms from the octahedral sites along the $[001]_{\mathrm{M}}$ axis, as mentioned in the previous paper $^{(5)}$. Therefore, the results shown in Fig. 2 indicate that the amount of getting away carbon atoms is a little in the earlier period of tempering while it is large in the latter period of tempering. The $a$ and $c$ parameters of the martensites tempered for more than $25.2 \mathrm{ks}$ at $358 \mathrm{~K}$ become difficult to be distinguished, as seen in Fig. 1(d). Therefore, the $a$ and $c$ parameters and $c / a$ ratios after the $259.2 \mathrm{ks} \mathrm{(3}$ days) tempering at $358 \mathrm{~K}$ were estimated with the aid of the data by Lyssak et al. ${ }^{(25)}$ on similar $\mathrm{Fe}-\mathrm{Mn}-\mathrm{C}$ alloys, and the dotted lines 
in Fig. 2 were drawn by using the parameters. The parameters after the $259.2 \mathrm{ks}$ at $358 \mathrm{~K}$ tempering are very similar to those at $428 \mathrm{~K}$ irrespective of the alloy content, in accordance with the Lyssak et al.'s data.

The two kinds of lattice parameter changes observed in Fig. 2 are supposed to correspond to those of the two stages, the preliminary and the first stages respectively, in the former definition that the tempering process has been divided into three stages in addition to the preliminary one. Therefore, the earlier and latter changes in the present tempering process will hereafter be referred to corresponding to the preliminary and the first stages, respectively.

\section{Electron microscopy of carbide formation}

\section{(1) Preliminary stage}

Photograph 1(a) is a transmission electron micrograph of a martensite in Specimen A tempered at $358 \mathrm{~K}$ for $3.6 \mathrm{ks}$. There are seen dislocation lines and short stripes along two directions as indicated with $\mathrm{A}$ and $\mathrm{B}$. The short stripes are more clearly seen from an enlargement, (d), of the framed area in (a). (b) and (c) are selected area electron diffraction patterns of the encircled area in (a), but (c) was taken after the specimen had slightly been tilted from the orientation exhibiting (b). As can be seen from the patterns, the specimen normal is nearly parallel to the $[010]_{M}$ (suffix $M$ means the martensite lattice), as explained in (e). It should be noticed that martensite reflection spots in both the patterns are distorted into a rectangular shape, and that split superlattice reflections appear between the incident and $00 \overline{2}$ reflection in (b), although they are fairly diffuse. From previous works on the low temperature tempering of $\mathrm{Fe}-\mathrm{Cr}-\mathrm{C}^{(17)}$ and $\mathrm{Fe}-$ $C^{(18)-(20)}$ alloys, the distortion of martensite reflection spots can be supposed to be caused by longer exposure ( $15 \mathrm{~s}$ with X-ray film) of satellite reflections and/or streaks around the martensite reflections. The appearance of satellite reflections and streaks have been attributed to the formation of a modulated structure $^{(18)-(20)}$ and a short range order of carbon atoms ${ }^{(26)(27)}$, respectively. The short stripes observed along the two directions in (a)
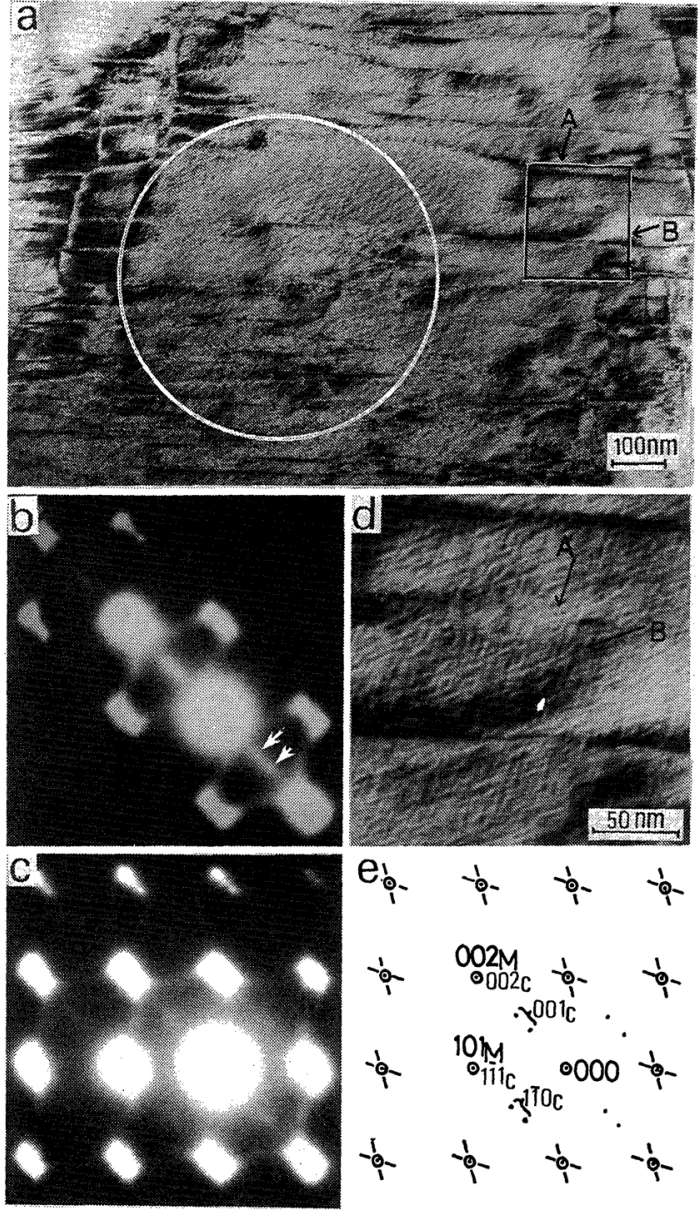

Photo. 1 Electron micrograph of a martensite, (a), and the corresponding electron diffraction patterns, (b) and (c), taken from the encircled area in (a), which were taken from $\mathrm{Fe}-4.1$ mass $\% \mathrm{Mn}-1.25$ mass $\% \mathrm{C}$ alloy tempered at $358 \mathrm{~K}$ for $3.6 \mathrm{ks}$. (d) is an enlargement of the framed area in (a), and (e) is a key diagram to the diffraction patterns.

and (d) may correspond to such a modulation and/or a short range ordering, because an angle made by the two directions is about $57^{\circ}$ and this is nearly equal to that made by the two directions of streaks.

The appearance of split superlattice reflections may be explained by the formation of a long period ordered tetragonal phase (reflection from this phase will be shown with suffir $\mathrm{C}$ in the key diagram), which is a modificatior of the $\mathrm{Fe}_{4} \mathrm{C}$ carbide, as identified by Nagakurc et al. ${ }^{(18)-(20)}$ in the $\mathrm{Fe}-\mathrm{C}$ alloys. Therefore the preliminary stage can be considered tr 
correspond to the formation of a modulated structure and/or a short range order of carbon atoms and a long period ordered tetragonal phase. As Nagakura et al. pointed out, the long period ordered tetragonal phase is so unstable that the split superlattice reflections can no longer be distinguished in (c), which was taken after electron irradiation for a time.

Photograph 2(a) is another electron micrograph showing a martensite of the same alloy as in Photo. 1. There are also seen dislocation lines, and the structure is very similar to that of the as-transformed martensite. However, in (b) which is a corresponding diffraction pattern taken from the whole area in (a), many extra reflections are seen other than martensite reflections of $[031]_{M}$ zone. The martensite reflection spots are again distorted a little into a rectangular shape as in Photos. 1(b) and (c), indicating the formation of a modulated structure and/or a short range order of carbon atoms. Of the extra reflections, the ones indicated with a mark $(\triangle)$ are due to the martensite reflections in another reciprocal plane and appear by some specimen buckling, as known later more clearly. The other extra reflections can be identified to be due to the previous long period ordered tetragonal phase $(\uparrow)$ and another carbide $(\uparrow)$, as explained in (c). As will be verified later, the other carbide is the one characteristic of the first tempering stage, and can be identified to be " $\eta$-carbide" (reflections from this carbide are shown with no suffix in the key diagram). Tempering for 3.6 $\mathrm{ks}$ at $358 \mathrm{~K}$ is in between the preliminary and first stages, as known from Fig. 2. Therefore, in the area exhibiting Photo. 2(a), there seem to appear two phenomena characteristic of the preliminary stage (the formation of a modulated structure and/or a short range order and a long period ordered tetragonal phase) and the first stage (the formation of $\eta$-carbide).

\section{(2) First stage}

Photograph 3(a) is an electron micrograph showing a martensite in Specimen A tempered at $428 \mathrm{~K}$ for $300 \mathrm{~s}$, corresponding to the first stage. A mottled pattern is observed, which seems to be caused by the precipitation of carbides. In fact, the carbides are recongnized

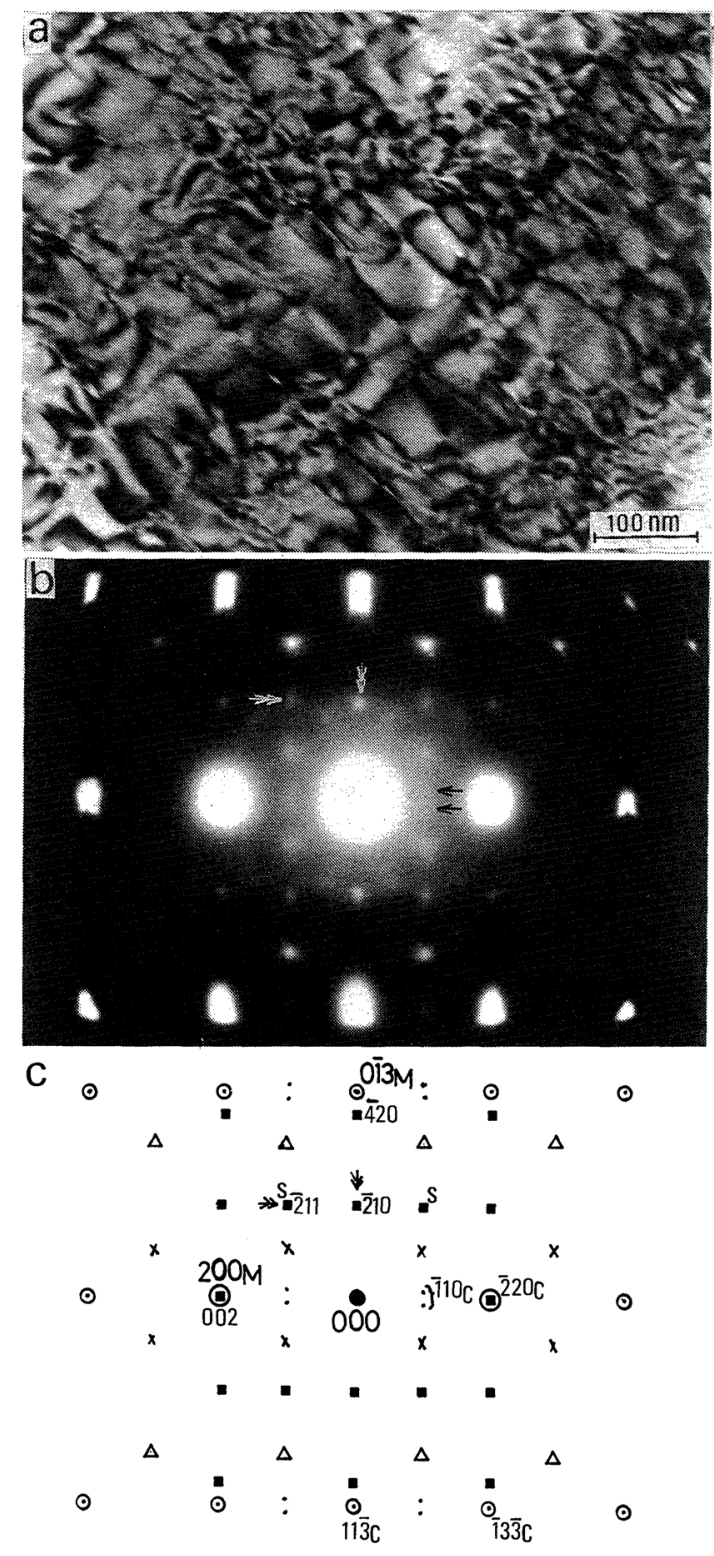

Photo. 2 Electron micrograph of a martensite, (a), and the corresponding diffraction pattern, (b), taken from the whole area of (a), which were taken from another area of the same specimen as in Photo. 1 , (c) being a key diagram to the diffraction pattern.

as bright spots in (b) which is a dark field image taken by using a reflection other than martensite reflections. The reflection used is the one marked with a circle in the corresponding diffraction pattern, (c), taken from the bright circle area in (a). (d) and (e) are electron diffraction patterns of the same area as for (c), but 

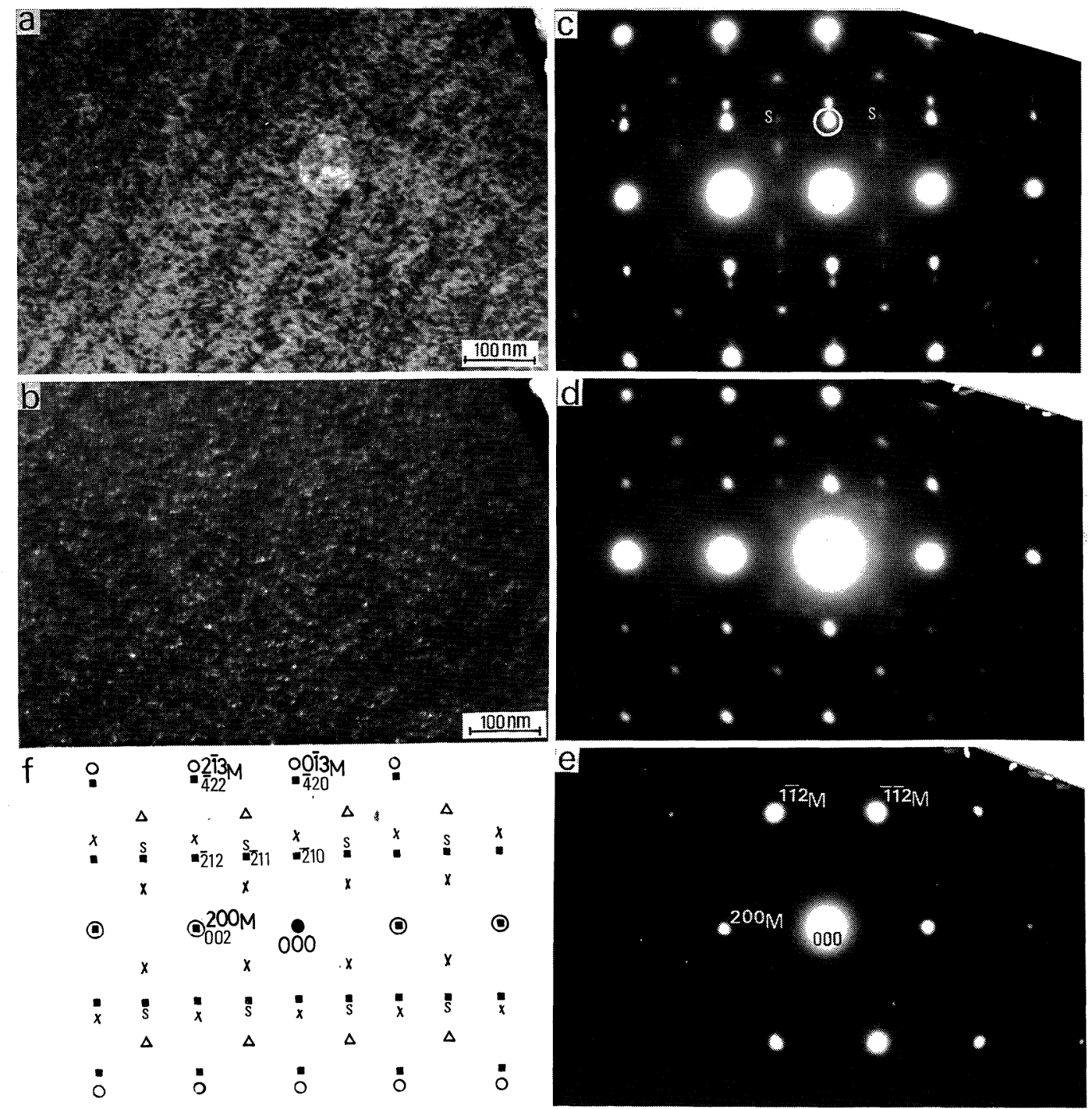

Photo. 3 Electron micrograph of a martensite, (a), its dark field micrograph taken by using $\overline{2} 10 \eta$-carbide reflection, (b), and a series of electron diffraction patterns, (c), (d) and (e), taken by changing the specimen orientation from the same area in (a), which were taken from $\mathrm{Fe}-4.1$ mass $\% \mathrm{Mn}-1.25$ mass $\% \mathrm{C}$ alloy tempered at $428 \mathrm{~K}$ for $0.3 \mathrm{ks}$. (f) is a key diagram to the pattern, (c).

they were taken by changing the specimen orientation. From the patterns, the incident electron beams are known to be nearly parallel

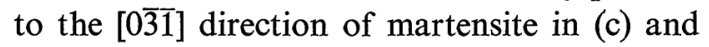
(d), and to the $[0 \overline{2} \overline{1}]$ direction in (e). Thus, some reflections in (c) are explained as superposition to $[0 \overline{3} \overline{1}],(\bigcirc)$, and $[0 \overline{2} \overline{1}],(\triangle)$, zone patterns, Photo. 2(b) being in a similar situation. Other extra reflections in (c) are from a carbide ( $\mathbf{v})$ and due to double diffractions $(x)$ between the martensite and carbide reflections. In order to identify the crystal structure of the carbide, spacings of planes producing the carbide reflections were measured by using those producing the martensite reflections as an internal standard, the martensite lattice parameters in Fig. 2 being referred. As a result, the carbide reflections were indexed to be of the [120] zone pattern of the $\eta$-carbide identified by Hirotsu et al. ${ }^{(21)}$ for $\mathrm{Fe}-\mathrm{C}$ alloys, as ex- 
plained in (f). As a reflection of the higher carbon content in the alloy, the superlattice reflections due to the carbon atom ordering are observed at the position marked with $s$. Then, the present carbide in the first tempering stage will also be referred to the $\eta$-carbide, and the space group will be represented with Pnnm. It can thus be concluded from Photo. 3 that the first tempering stage is characterized by the precipitation of the $\eta$-carbide.

Photographs 4(a) and (b) are electron diffraction patterns taken from Specimen B tempered at $428 \mathrm{~K}$ for $3.6 \mathrm{ks}$, which have been taken to indicate more clearly the orthorhombic nature of the carbide. In (a) are seen a $\langle 110\rangle$ zone pattern, $(\bigcirc)$, and its twin pattern, $(\bigcirc)$, from the martensite and the [010] zone pattern; $(\boldsymbol{\square})$, from the $\eta$-carbide (the mark $\times$ represents a spot due to double diffraction between the martensite twins). The $\langle 110\rangle$ zone pattern is uniquely indexed to be of the [011] zone's because the ratio of A-B distance to C-D distance is 1.42 , and this is in agreement with the theoretical one, 1.420 , calculated from the corresponding lattice parameters in Fig. 2. In the [010] zone pattern from the $\eta$-carbide, the ratio of E-F distance to F-G distance is 1.62 , showing clearly the orthorhombic distortion of the $\eta$-carbide. In (b) are seen a $\langle 001\rangle$ zone pattern from martensite and the [001] zone pattern from the $\eta$-carbide. The superlattice reflections (s) due to the carbon atom ordering are seen with very weak intensity reflecting the lower carbon content. The $\langle 001\rangle$ zone martensite pattern can uniquely be indexed to be of the [100] zone's from a similar analysis to the above and a trace analysis of transformation twins in a corresponding electron micrograph. Other various zone patterns as well as the previous ones (Photos. 3 and 4) were analyzed using martensite reflections as an internal standard, and the lattice parameters $(\mathrm{nm})$ of the $\eta$-carbide were determined as follows;

$$
a=0.470, b=0.429 \text { and } c=0.285 \text {. }
$$

These parameters are nearly the same as those for the orthorhombic lattice determined by Hirotsu et al. and Shimizu et al. for $\mathrm{Fe}-\mathrm{C}$ alloys, and also the same for both the Specimens $A$ and $B$ if they are tempered up to the first
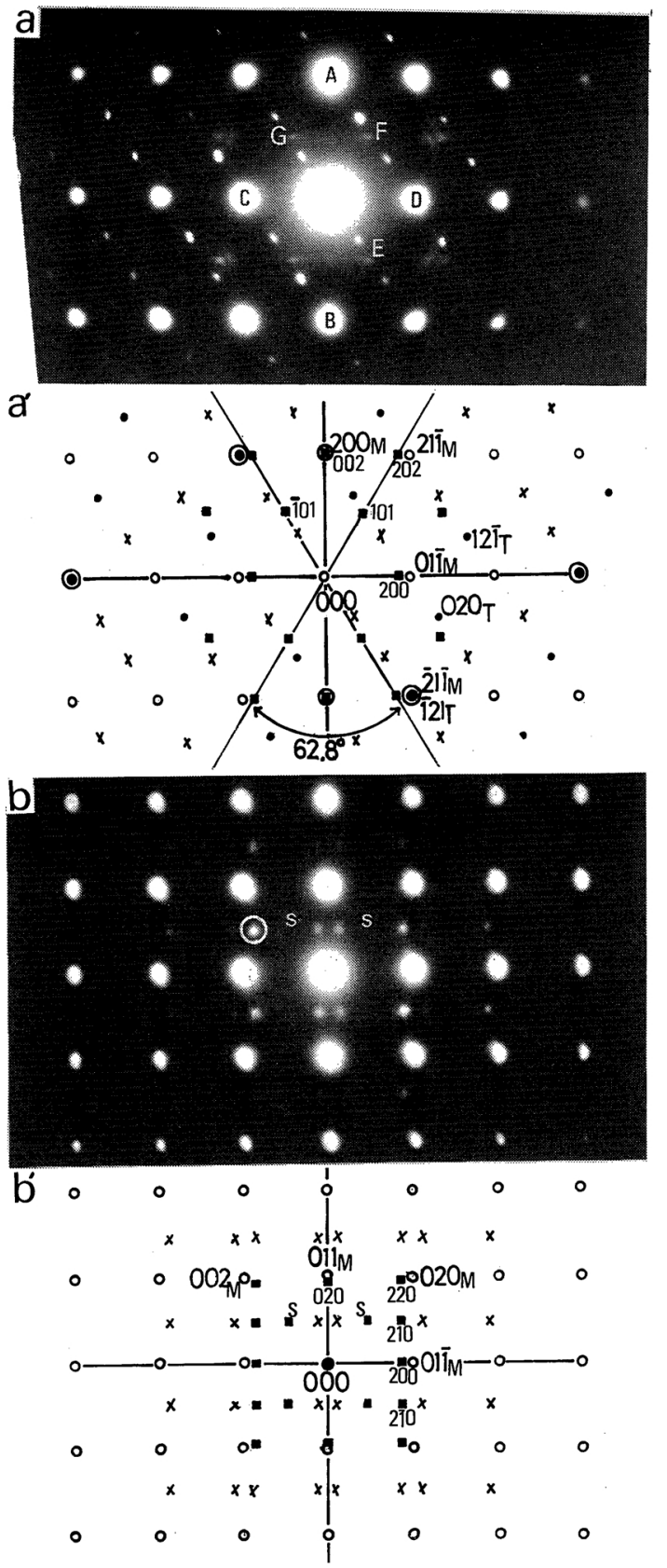

Photo. 4 Electron diffraction patterns taken from $\mathrm{Fe}-5.2$ mass $\% \mathrm{Mn}-0.93$ mass $\% \mathrm{C}$ alloy tempered at $428 \mathrm{~K}$ for $3.6 \mathrm{ks}$, showing the [011] martensite and [010] carbide zone patterns, (a), and the [100] martensite and [001] carbide zone patterns, (b), $\left(a^{\prime}\right)$ and $\left(b^{\prime}\right)$ being key diagrams to (a) and (b), respectively. 
stage. According to the parameters determined, an angle made by the $\overline{1} 01$ and 101 reciprocal vectors of the carbide must be $62.3^{\circ}(1.0873$ rad.), and this is in good agreement with the measured one, $62.8^{\circ}$ (1.0961 rad.), in Photo. 4(a).

\section{(3) Morphology of the carbide and orientation relationship between the carbide and martensite lattices}

Photograph 5(a) is an electron micrograph of the martensite from which Photo. 4(b) was taken. There are seen internal transformation twins and carbide precipitates, although the contrast of carbides in almost all the area is not clear because of the simultaneous reflections from the carbide and martensite crystals. The carbides seen in the lower left corner appear to

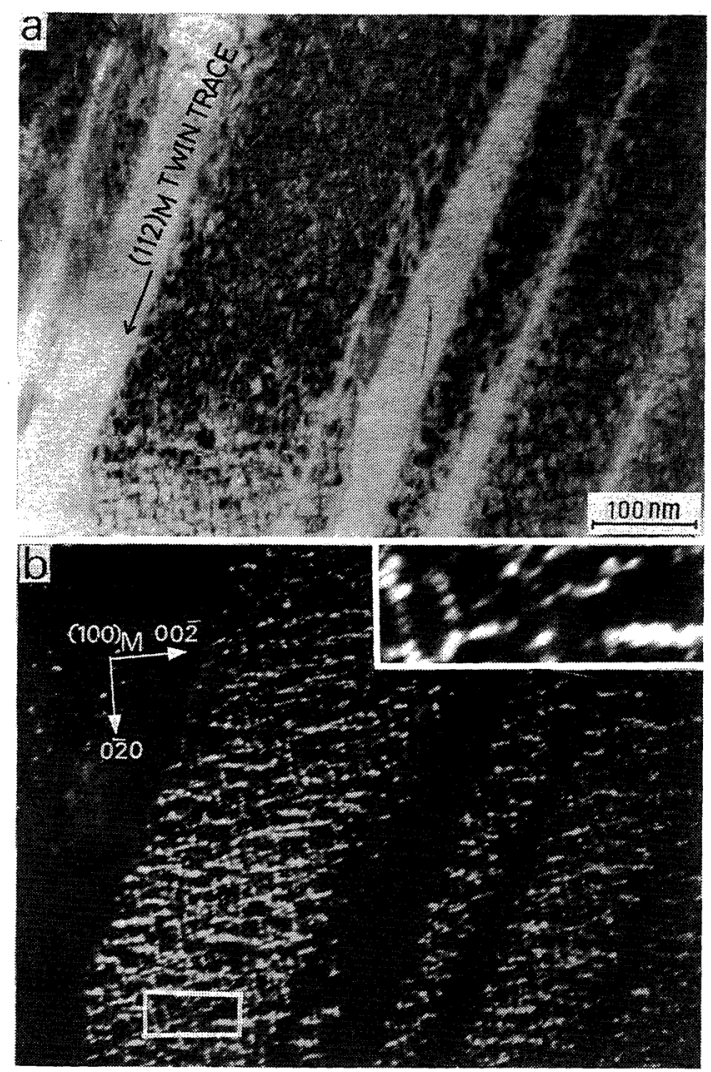

Photo. 5 Electron micrograph of the martensite from which Photo. 4 (b) was taken, (a), and its dark field micrograph, (b), taken by using $\overline{2} 10 \eta$-carbide reflection. The upper right corner is an enlargement of the framed area in (b). have a rod or a plate shape along the $[010]_{M}$ and $[001]_{M}$ directions. However, in the dark field image, (b), taken by using the $\overline{2} 10 \eta$-carbide reflection in Photo. 4(b), the carbides along the two directions are clearly seen all over the area, and it can be seen that the apparent shape of rod or plate carbide consists of a row of finer carbide particles. In Photo. 3(b), the carbides were observed only as bright spots but not as a row of fine particles, presumably being due to a short tempering period. The carbide morphology consisting of fine particles is consistent with that observed by Hirotsu et al. and Shimizu et al. in $\mathrm{Fe}-\mathrm{C}$ alloys. In the present work, however, the arrangement of the carbide particles in a rod or plate is different depending on the row direction, that is, the largest areal plane of the carbide particles arranged along the $[010]_{M}$ direction is normal to that direction, while that along the $[001]_{M}$ direction is inclined by $45^{\circ}$ to that direction, as schematically shown in Fig. 3 and clearly seen from the enlargement inserted at the upper right corner. This result means that the carbide precipitation along the tetragonal axis of martensite is different from that along other principal axes.

Next, the orientation relationship between the carbide and the martensite lattices will be examined. In Photo. 4(a), 200 and 002 reciprocal vectors of the $\eta$-carbide are parallel to $01 \overline{1}$ and 200 reciprocal vectors of the martensite, respectively. In Photo. 4(b), 200

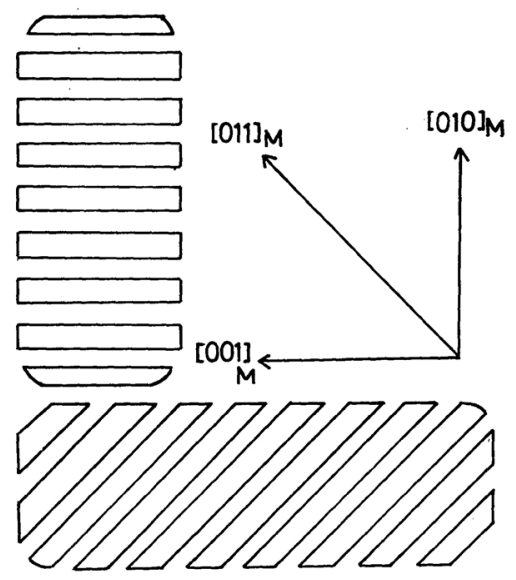

Fig. 3 Schematic illustration of the arrangements of fine carbide disks or ribbons forming an apparent rod or plate shape. 


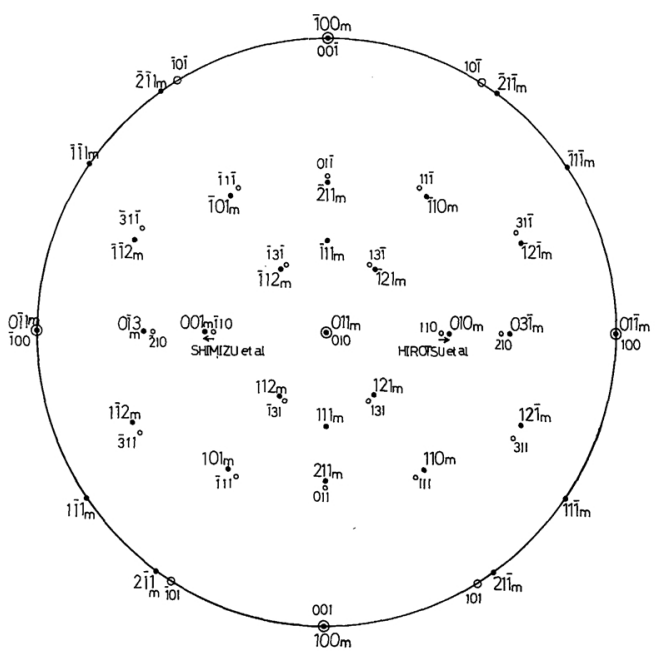

Fig. 4 Stereographic representation of the obtained orientation relationship between the $\eta$-carbide and martensite lattices, corelation with previous ones being also shown with the arrows.

and 020 reciprocal vectors of the $\eta$-carbide are parallel to $01 \overline{1}$ and 011 reciprocal vectors of the martensite, respectively. Putting the relations together, the following orientation relationships can be obtained;

$$
(011)_{\mathrm{M}} / /(010)_{\eta}
$$

and

$$
[100]_{\mathrm{M}} / /[001]_{\eta} \text {. }
$$

These relationships hold for both the specimens tempered at $428 \mathrm{~K}$, and they are also obtained for both the specimens tempered at $358 \mathrm{~K}$. Figure 4 is a stereographic representation of the above orientation relationships, in which corelations with the previous ones obtained by Hirotsu et al. and Shimizu et al. are also indicated. One of the previous two is obtained if the carbide lattice is rotated around the $[100]_{M}$ axis toward either direction of the arrows by the angle indicated with arrows, and the other is obtained if the similar operation is done toward the opposite direction.

\section{Discussion}

In the above, it has been shown that the carbon segregation and the carbide formation in $\mathrm{Fe}-\mathrm{Mn}-\mathrm{C}$ alloys proceed in almost the same manner as those in other $\mathrm{Fe}-\mathrm{C}$ and $\mathrm{Fe}-(\mathrm{Ni}$ or
$\mathrm{Cr}$ )-C alloys. However, some differences are noticed to exist between the present and previous results, as is discussed below.

The peak heights of the $\{110\}$ and $\{011\}$ martensite reflections in Fig. 1 are lower than those in Fig. 9 of Ref. (5), although strictly speaking such a comparison may not be in place since the incident condition of $\mathrm{X}$-rays is not necessarily the same in the present and previous experiments. The lower peak heights may be supposed to be due partly to a small amount of martensities. In the previous work, specimens were slowly cooled and heated in order to measure lattice parameters successively at various temperatures, and therefore isothermal martensites might be formed additionally when compared with the present specimens rapidly cooled to liquid nitrogen temperature and heated up to room temperature. Such a different amount of martensites brings about a difference in lattice parameters, as verified in the previous work, and actually the present lattice parameters were slightly different from the previous ones.

The split superlattice reflections in Photo. 1(b), which have been attributed to the formation of a long period ordered tetragonal phase, were so diffuse that it was not easy to distinguish as two reflection spots. On the other hand, they were fairly sharp and distinguishable in the diffraction patterns taken by Izotov et al. ${ }^{(17)}$ and Nagakura et al. ${ }^{(18)-(20)}$. Although exact origin of the diffuseness is not clear, it is supposed to be in a larger divergency of electron beams and in a smaller domain of the long period ordered tetragonal phase (possibly due to the smaller amount of carbon atoms), comparing with the previous works.

Figure 5 shows the atomic arrangement of iron atoms in the (010) $\eta$-carbide plane and the (011) martensite plane which are parallel to each other in the present orientation relationship. The atomic arrangements as well as spacings are very similar to each other and therefore the parallelism seems to be reasonable. However, the previous orientation relationships obtained by Hirotsu et al. and Shimizu et al. are not exclusive judging from a similarlity of the atomic spacings in their parallel planes. All the three orientation relationships may possibly 


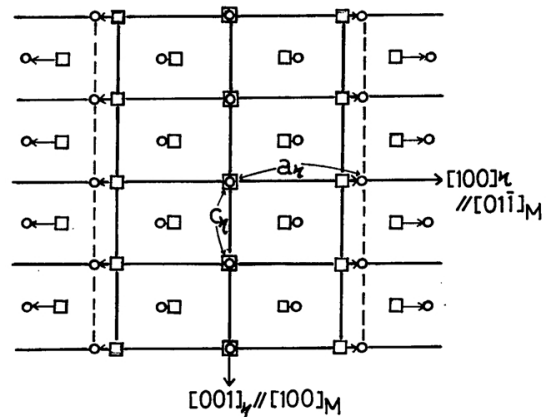

Fig. 5 Atomic arrangements in the (010) $\eta$-carbide plane and (011) martensite plane which are parallel to each other according to the orientation relationship.

exist, and either may predominantly be realized depending on a carbide morphology and/or a coherency strain between the carbide and martensite lattices. In the present work, two kinds of arrangements of disk or ribbon shape carbide particles have been observed, as schematically shown in Fig. 3. Rows of the carbide particles along the $[001]_{M}$ tetragonal axis occupy a large area in Photo. 5, and they might exhibit the present orientation relationships. Other rows along the $[010]_{M}$ or $[100]_{M}$ principal axis may exhibit either of the previous ones by Hirotsu et al. and Shimizu et al. The fact that the rows of carbide particles along the $[010]_{M}$ direction in Photo. 5 are very similar to those observed by the previous workers seems suggest a possibility of the realization of the previous orientation relationship. However, the rows of carbide particles along the $[010]_{M}$ direction were very few in Photo. 5, and so the previous orientation relationship would not be detected.

All the electron diffraction patterns have been taken from 0.1 to $0.6 \mu \mathrm{m}$ areas, but they exhibited only one zone pattern from the $\eta$ carbide as if only one orientation of the $\eta$-carbide exists in such areas. However, some other diffraction patterns exhibited two zone patterns from the $\eta$-carbide. The apparent one zone pattern may be due to a fact that one variant of the carbides satisfies the Bragg condition but another variant does not.

\section{Acknowledgements}

This work was partly supported by the
Grant-in-Aid for Fundamental Scientific Research (Ippan A, 1980-81) from the Ministry of Education, Science and Culture of Japan, and the support is greatly appreciated.

\section{REFERENCES}

(1) M. Oka, Y. Tanaka and K. Shimizu: Jap. J. Appl. Phys., 11 (1972), 1073.

(2) M. Oka, Y. Tanaka and K. Shimizu: Trans. JIM, 14 (1973), 148.

(3) K. Shimizu and Y. Tanaka: Trans. JIM, 19 (1978), 685.

(4) Y. Tanaka and K. Shimizu: Trans. JIM, 21 (1980), 34

(5) Y. Tanaka and K. Shimizu: Trans. JIM, 21 (1980), 42.

(6) Y. Tanaka and K. Shimizu: Trans. JIM, 22 (1981), 392.

(7) G. V. Kurdjumov and L. I. Lyssak: J. Iron Steel Inst., 156 (1947), 29.

(8) C. S. Roberts, B. L. Averback and M. Cohen: Trans. ASM, 45 (1953), 291.

(9) B. S. Lement, B. L. Averback and M. Cohen: Trans. ASM, 47 (1955), 291.

(10) B. S. Lement and M. Cohen: Acta Met., 4 (1956), 469.

(11) R. D. Heidenreich, L. Sturkey and H. L. Woods: J. Appl. Phys., 17 (1946), 127.

(12) K. H. Jack: J. Iron Steel Inst., 169 (1951), 26.

(13) M. P. Arbuzov and B. V. Khayenko: Fiz. Metal. Metalloved., 13 (1962), 128.

(14) M. G. H. Wells: Acta Met., 12 (1964), 389.

(15) E. Tekin and P. M. Kelly: Precipitation from Iron Base Alloys, Gordon and Breach (1965).

(16) G. J. Barton: Acta Met., 17 (1969), 1085.

(17) V. I. Izotov and L. M. Uteusky: Fiz. Metal. Metalloved., 25 (1968), 98.

(18) S. Nagakura and M. Toyoshima: Trans. JIM, 20 (1979), 100.

(19) S. Nagakura, K. Shiraishi and M. Toyoshima: Proc. 1st JIM Int. Symp. on New Aspects of Martensitic Transformation, Suppl. to Trans. JIM, 17 (1976), 299.

(20) M. Toyoshima and S. Nagakura: Modulated Structures (AIP Conf. Proc. No. 53) (1979), 263.

(21) Y. Hirotsu and S. Nagakura: Acta Met., 20 (1972), 645.

(22) Y. Hirotsu and S. Nagakura: Trans. JIM, 15 (1974), 129.

(23) Y. Hirotsu, Y. Itakura, K. C. Su and S. Nagakura: Trans. JIM, 17 (1976), 503.

(24) K. Shimizu and H. Okamoto: Trans. JIM, 15 (1974), 193.

(25) L. I. Lyssak and Yu. M. Polishchul: Fiz. Metal. Metalloved., 27 (1969), 148.

(26) A. G. Khachaturyan and T. A. Onosimova: Fiz. Metal. Metalloved., 26 (168), 973.

(27) S. Nagakura, K. Shiraishi and Y. Hirotsu: Trans. JIM, 16 (1975), 601. 